\title{
Confining electrodeposition of metals in structured electrolytes
}

\author{
Snehashis Choudhurya, ${ }^{\mathrm{a}, 1}$, Duylinh Vu, ${ }^{\mathrm{a}, 1}$, Alexander Warren ${ }^{\mathrm{a}}$, Mukul D. Tikekar ${ }^{\mathrm{b}}$, Zhengyuan Tu', and Lynden A. Archer,

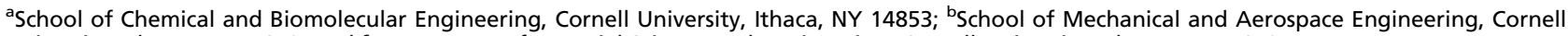 \\ University, Ithaca, NY 14853; and 'Department of Material Science and Engineering, Cornell University, Ithaca, NY 14853
}

Edited by Thomas E. Mallouk, The Pennsylvania State University, University Park, PA, and approved May 16, 2018 (received for review February 24, 2018)

Electrochemical cells based on alkali metal ( $\mathrm{Li}, \mathrm{Na}$ ) anodes have attracted significant recent attention because of their promise for producing large increases in gravimetric energy density for energy storage in batteries. To facilitate stable, long-term operation of such cells a variety of structured electrolytes have been designed in different physical forms, ranging from soft polymer gels to hard ceramics, including nanoporous versions of these ceramics that host a liquid or molten polymer in their pores. In almost every case, the electrolytes are reported to be substantially more effective than anticipated by early theories in improving uniformity of deposition and lifetime of the metal anode. These observations have been speculated to reflect the effect of electrolyte structure in regulating ion transport to the metal electrolyte interface, thereby stabilizing metal electrodeposition processes at the anode. Here we create and study model structured electrolytes composed of covalently linked polymer grafted nanoparticles that host a liquid electrolyte in the pores. The electrolytes exist as freestanding membranes with effective pore size that can be systematically manipulated through straightforward control of the volume fraction of the nanoparticles. By means of physical analysis and direct visualization experiments we report that at current densities approaching the diffusion limit, there is a clear transition from unstable to stable electrodeposition at Li metal electrodes in membranes with average pore sizes below $500 \mathrm{~nm}$. We show that this transition is consistent with expectations from a recent theoretical analysis that takes into account local coupling between stress and ion transport at metalelectrolyte interfaces.

electrodeposition | confinement | dendrites | batteries | nanoparticles

$\mathbf{R}$ echargeable electrochemical cells based on alkali metal anodes provide opportunities to substantially push the energy storage limits of batteries. Such cells achieve this feat both by increasing the amount of electrical energy that can be stored on a mass or volume basis at the anode and by enabling more flexible material choices for the cathode (1-6). Parasitic reactions between the chemically reactive anode (7-11) and proliferation of rough, dendritic/mossy electrodeposition at the metal anode during battery recharge have been reported to reduce stability of the cells by increasing the likelihood of failure by internal short circuits and by increasing the surface area of reactive metals in contact with electrolytes $(12,13)$.

The stability analysis of electrodeposition reported by Monroe and Newman $(14,15)(\mathrm{MN})$ employs continuum modeling of ion transport processes and mechanics in bulk electrolytes to predict morphological transitions at a metal anode. This work showed that marginal stability is achieved at any current density in an electrolyte with mechanical modulus about twice that of the metal electrode. One study using block copolymer electrolytes based on hard polystyrene and ion conductive poly(ethylene oxide) segments to systematically adjust the electrolyte modulus provided early support for this prediction (16-19). This finding, however, stands in contrast to a large and growing body of recent experimental reports which show that rough deposition of metals can be inhibited in gel-like electrolytes $(20,21)$, crosslinked polymer electrolytes $(18,22,23)$, and in ion-conducting polymer electrolytes (24-26), all with mechanical moduli three or more orders of magnitude below that of the metal electrode. Recently, Zhao et al. (27) showed that a composite polymer electrolyte with immobilized ions and ceramic fillers can indeed inhibit dendrites at ambient conditions. Even more dramatic are reports which show that dendrite-induced short circuits in lithium and sodium batteries can be delayed or even eliminated at low and moderate current densities in liquid electrolytes in which a fixed fraction of anions is maintained at the interface (28-33), or in liquid electrolytes in which halide ions are present at the electrodeelectrolyte interface $(11,34-37)$. Essentially nothing is known about the working mechanisms by which such low-modulus materials prevent metal dendrite proliferation at either low or high current densities, relative to the diffusion-limiting current $J^{*}$, and a variety of qualitative arguments have been proposed to explain their working mechanism.

The present study is motivated by recent theoretical work by Tikekar et al. $(7,38)$, which extended the MN analysis to consider the effect of local stress-transport coupling with ion migration profiles and the impact such coupling has on stability of metal electrodeposition. This analysis shows that at current densities below $J *$, stable electrodeposition of metals can be achieved under a broad range of conditions, including in electrolytes with shear moduli as low as $0.1 \mathrm{MPa}$ (i.e., more than four orders of magnitude lower than that of the metal electrode). Another conclusion from the analysis is that electrolytes created by hosting simple liquids in porous materials should be able to

\section{Significance}

Electrochemical cells based on lithium and sodium metal anodes are considered among the most versatile platforms for highenergy electrical energy storage. Unfortunately, unstable dendritic metal deposition at currents below the diffusion limit lead to premature cell failure by internal short circuits. In a volatile electrolyte, the ohmic heat generated by these shorts pose significant safety risks. The article reports that electrodeposition of metals can be stabilized by confining ion transport to length scales below a few hundred nanometers. It is also shown that dendrite growth can be arrested in electrolytes with mechanical moduli well below that of the metal. This finding contradicts current orthodoxy, which holds that solid-state electrolytes with moduli higher than the metal are required for preventing dendrite growth.

Author contributions: S.C., Z.T., and L.A.A. designed research; S.C., D.V., and A.W. performed research; S.C., D.V., A.W., M.D.T., Z.T., and L.A.A. analyzed data; and S.C., D.V., and L.A.A. wrote the paper.

The authors declare no conflict of interest.

This article is a PNAS Direct Submission.

Published under the PNAS license.

${ }^{1}$ S.C. and D.V. contributed equally to this work.

${ }^{2}$ To whom correspondence should be addressed. Email: laa25@cornell.edu.

This article contains supporting information online at www.pnas.org/lookup/suppl/doi:10 1073/pnas.1803385115/-/DCSupplemental.

Published online June 11, 2018. 
prevent rough electrodeposition of any metal when the average pore size of the host falls below a certain critical electrolytedependent value. This prediction is important because it means that solid-state electrolytes are not required to stabilize electrodeposition at metal anodes in batteries; it is also testable. Here, we design structured electrolytes based on cross-linked nanoparticle-polymer hybrid membranes in which the effective pore size can be facilely manipulated by changing the volume fraction of particles in the precursor material. We also use these electrolytes to systematically investigate the effect of electrolyte network structure and mechanical properties on the stability of metal deposition at currents close to the critical current density, $J^{*}$. The study takes advantage of an optical visualization technique that allows time-dependent changes in morphology of the metal-electrolyte interface to be directly imaged. Used in combination with the analysis of Tikekar et al. (38), these efforts are shown to lead to a comprehensive understanding of how and why structured electrolytes that do not meet the MN modulus criteria are able to prevent metal dendrite proliferation.

Fig. $1 A$ shows the synthetic route used to create cross-linked hairy nanoparticle-based electrolytes. Briefly, silica nanoparticles (diameter $\sim 10 \mathrm{~nm}$ ) are first grafted in aqueous solution with an alkoxy silane terminated oligomeric polyethylene glycol (PEO$\left.\mathrm{OH}, M_{\mathrm{w}}=500 \mathrm{~g} / \mathrm{mol}\right)$. As shown in our previous work $(18,39$, $40)$, the approach produces $\mathrm{SiO}_{2}$ nanoparticles densely grafted $\left(\sum \sim 1\right.$ chain per $\mathrm{nm}^{2}$; from thermal gravimetric analysis) with PEO chains bearing a reactive terminal hydroxyl group. The resultant $\mathrm{SiO}_{2}-\mathrm{PEO}-\mathrm{OH}$ nanoparticles are subsequently used as multifunctional node points for cross-linking poly(propylene oxide) (PPO, $M_{\mathrm{w}}=2,000 \mathrm{~g} / \mathrm{mol}$ ) chains functionalized with isocyanate groups at both ends. A straightforward calculation shows that on average there are $\sim 300$ PEO-OH molecules anchored to a single $\mathrm{SiO}_{2}$ nanoparticle, meaning that materials with a high degree of cross-linking are achieved. Additionally, because the PEO chains are short and the PPO linkers are amorphous at ambient temperatures, the cross-linked materials exist as freestanding membranes with high flexibility and toughness for silica content of $6.5 \%$ (by weight) (Fig. $1 B$ ). The large number of grafting sites available on each $\mathrm{SiO}_{2}$ nanoparticle makes it possible to introduce different functionalities, including ionic species (41) or flame retardants such as phosphates (42) in the materials. Cross-linking these nanostructures provides a facile route to nanoporous membranes in which the surface chemistry of the pores can be manipulated for regulating the ion transport characteristics or for preventing thermal runway in lithium

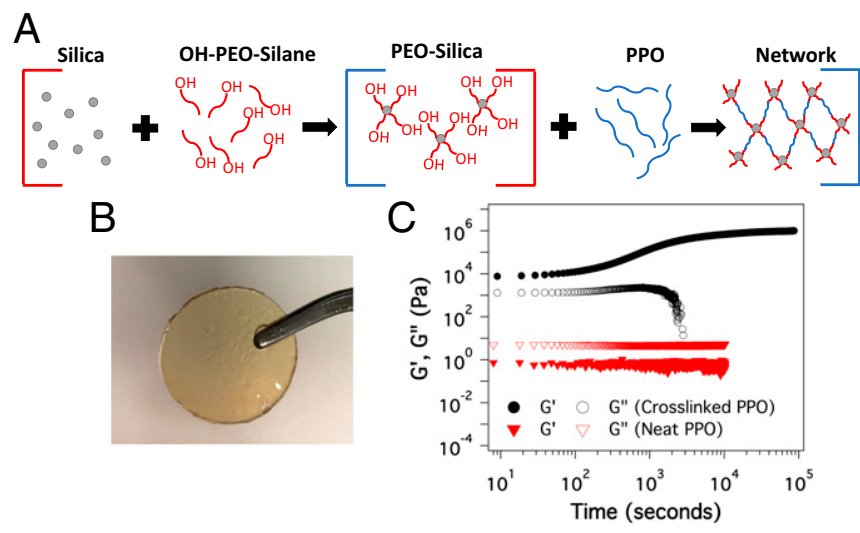

Fig. 1. Synthesis cross-linked hairy nanoparticles. (A) Schematic showing synthesis procedure of the cross-linked nanoparticles. (B) Photograph of the freestanding membrane with $6.8 \%$ (by weight) silica content. (C) Timesweep measurements under oscillatory shear at strain $1 \%$ and frequency of $10 \mathrm{~Hz}$ at $60^{\circ} \mathrm{C}$. batteries. Furthermore, by varying the volume fraction of particles it is possible to systematically change the configuration of the particle-tethered PEO and PPO chains in the cross-linked materials to create membranes with vastly different pore sizes and mechanical properties.

It is possible to monitor the progress of the cross-linking reaction by measuring the time-dependent development of elasticity in the materials by means of dynamic shear rheological measurements (Fig. 1C). Specifically, we employed oscillatory shear to continuously measure the storage and loss modulus of the initial slurry of PEO-grafted nanoparticles and PPOdiisocyanate chains at $60{ }^{\circ} \mathrm{C}$ using a small-amplitude shear strain of $1 \%$ and a fixed oscillatory shear frequency of $10 \mathrm{~Hz}$. It is observed that the storage modulus increases over time indicative of the cross-linking process, which reaches its maximum value in about $24 \mathrm{~h}$. It is important to note that although the chemical reaction between an isocyanate and hydroxyl group is very fast, the overall cross-linking reaction kinetics are under diffusion control as it becomes progressively harder for PPO chains tethered to one particle to bond to a neighboring site on another particle to increase network elasticity. It can also be seen from Fig. $1 C$ that the loss modulus decreases and rolls over to very low values at long times, consistent with the gradual loss of dissipation processes as the network becomes more fully cross-linked. These effects are most dramatically demonstrated by comparison of the storage and loss moduli for the unlinked PPO polymer. It is seen that whereas the loss modulus dominates storage for the unlinked, liquid-like material, the storage modulus dominates as cross-linking nears completion. It is remarkable that at just $6.8 \mathrm{wt}$ $\%$ of $\mathrm{SiO}_{2}$ in the materials there is increment of over five orders of magnitude in modulus.

More in-depth knowledge of the configuration and dynamics of the linker polymer chains is possible from dielectric relaxation measurements. It is known that polymers such as PPO and cis-1,4polyisoprene possess type-A dipole that facilitate alignment of the polymer chain end-to-end vector in the direction of an imposed electric field; thus, one can quantify the end-to-end chain relaxation from the dielectric loss spectrum $(43,44)$. Additionally, from the magnitude of the loss peak, one can calculate the dielectric strength, which for flexible, amorphous chains can be used to estimate the relative degree of chain stretch (45). SI Appendix, Fig. S1 $A$ and $B$ shows the loss permittivity of the neat PPO and cross-linked PPO at various frequencies and temperatures. The experimental data were fitted using the Havriliak-Negami (H-N) equation: $\varepsilon^{*}(\omega)=\varepsilon_{\infty}+\Delta \varepsilon /\left[\left(1+(\mathrm{i} \omega \tau)^{\alpha}\right)^{\beta}\right]$, where $\alpha$ and $\beta$ are associated with broadening dielectric peaks, $\tau$ represents the $\mathrm{H}-\mathrm{N}$ relaxation time, $\omega$ is frequency, $\varepsilon_{\infty}$ is dielectric constant $\left(\varepsilon^{\prime}\right)$ at the high- frequency limit, and $\Delta \varepsilon$ is the dielectric strength. The relaxation times at various temperature obtained from the $\mathrm{H}-\mathrm{N}$ fits are shown in SI Appendix, Fig. S1C. It can be seen that the relaxation time for all temperatures follow the Vogel-FulcherTammann (VFT) model, represented by the continuous lines in the figure. The VFT model is given as $\tau=A \exp \left(-B / k\left(T-T_{0}\right)\right)$, where $A$ is the prefactor, $B$ is the activation energy, and $T_{o}$ is reference temperature, shown in SI Appendix, Table S1. It can be seen that the activation energy of the relaxation dynamics of PPO polymer increases dramatically from $9.8 \mathrm{~kJ} /$ mole to $20 \mathrm{~kJ} / \mathrm{mole}$ as a result of cross-linking, which is consistent with expectations based on the molecular weight of the polymers and high levels of cross-linking achieved (18). Comparison of the dielectric strength for the untethered and tethered PPO indicates that the linkers are also highly stretched (shown in SI Appendix, Fig. S1D). For random coil type-A dielectric polymer chains, the dielectric strength $\Delta \varepsilon$ is proportional to the mean squared end-to-end vector of the polymer chain. The results in SI Appendix, Fig. S1D show that $\Delta \varepsilon$ measured using the cross-linked membranes is 250 times larger than the corresponding value for the linear unlinked polymer. Considering that the molecular weight of the PPO chains is just 
$2 \mathrm{kDa}$, the large increases in $\Delta \varepsilon$ also imply that dipoles on the particle tethered chains are also highly correlated.

The cross-linked hairy nanoparticles provide an opportunity to understand the underlying physics of metal electrodeposition in heterogeneous environment, because these materials serve as model nanoporous structures such that the silica nanoparticles act as nonconductive physical barriers and the polymeric chains in the interparticle gaps as pathways for ion migration. In this context, the volume fraction of silica nanoparticles in the entire composite was varied and the corresponding interparticle distance was estimated by assuming random closed packing (r.c.p.) arrangement of spherical nanoparticles: $d_{p-p}=d\left[(0.63 / \Phi)^{1 / 3}-1\right]$, as reported in SI Appendix, Table S2 for different weight fraction of silica content. We utilized transmission electron microscope (TEM) to analyze the spatial arrangement of the nanoparticles in the polymer composite as shown in SI Appendix, Fig. S2A for r.c.p. pore sizes of 20,100, and $500 \mathrm{~nm}$. The interparticle distance of the silica nanoparticles was further measured from the TEM images and the corresponding variation was fitted to a normal distribution function obtained from the respective mean and SDs and plotted as an inset of the images. The average interparticle distances measured from TEM are observed to be similar to r.c.p. pore sizes (Fig. $2 A$ ). However, the variance of the distribution is seen to increase with decreasing silica volume fractions (SI Appendix, Fig. S3). We further performed frequency sweep measurements at a constant amplitude of $5 \%$ for different cross-linked PPO as well as neat PPO polymer. The storage and loss modulus of obtained from the measurements are plotted in SI Appendix, Fig. S2 $B$ and $C$, respectively. The results show that the composite materials at all cross-linking densities have higher $\mathrm{G}^{\prime}$ than $\mathrm{G}^{\prime \prime}$, indicating elastic behavior that is unseen at such low core volume fractions in
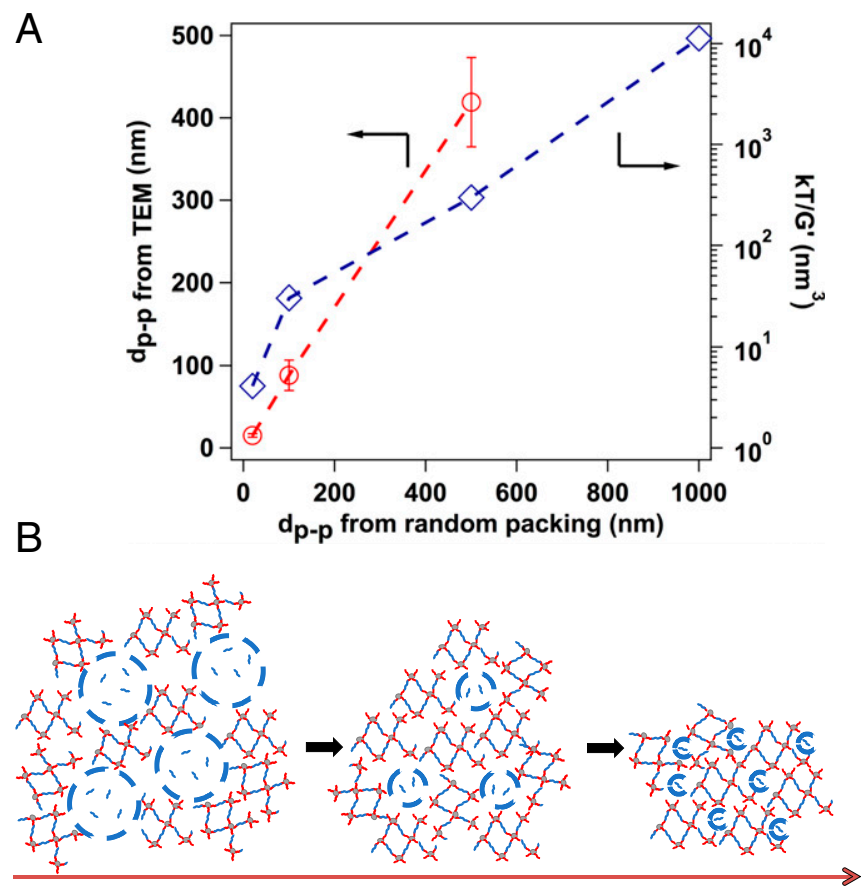

Increasing silica concentration in polymer network

Fig. 2. Characterization of pore architecture in the cross-linked structure. (A) Comparing the pore sizes obtained from TEM as well as cage sizes obtained from frequency-sweep measurements with the r.c.p. pore sizes. $(B)$ Schematic showing the changes in pore structures with the variation of silica nanoparticle volume fractions. In all of the subparts, the sample names are characterized by the r.c.p. interparticle distance between silica nanoparticles in the composites. colloidal suspensions. The elastic modulus is seen to progressively increase with increasing core volume fraction; the associated cage volume $\left(k T / G^{\prime}\right)$ is reported in Fig. $2 A$. Membranes with r.c.p. pore size $20 \mathrm{~nm}$ show the highest shear modulus of $\sim 1 \mathrm{MPa}$, while in the uncross-linked state the materials are simple Newtonian liquid. The higher variance of spatial distribution of silica nanoparticles and reminiscence of elasticity at low core fractions indicate that the particles form hierarchical string-like mass fractals due to the fixed length of the cross-linker (PPO), as shown in Fig. $2 B$, also confirmed using small-angle X-ray scattering in our previous work (18)

Next, we systematically study the morphology of the lithiumelectrolyte interface in the cross-linked hairy nanoparticles soaked with liquid electrolyte using direct visualization of the lithium metal anode during electrodeposition in an optical cell. The cell comprises a lithium metal and stainless-steel electrodes separated by a chamber containing electrolyte of $1 \mathrm{M} \mathrm{LiPF}_{6}-\mathrm{EC} /$ DMC (10). For the conditions used in the experiments, the diffusion-limited current density for the different membranes were calculated from the measured conductivity and transference number: $J^{*}=2 z c_{o} F D_{a p p}\left(t_{a} L\right)^{-1}$. Here, $z$ is the charge number of cation, $F$ is the Faraday constant, $c_{o}$ is salt concentration, $t_{a}$ is the anion transference number, and $\mathrm{L}$ is the interelectrode spacing $(1 \mathrm{~mm})$ and $D_{a p p}$ is the diffusion coefficient. Using the Bruce-Vincent method (33), the transference number (SI Appendix, Fig. S4) was determined to be 0.38 for the cross-linked nanoparticles (r.c.p. $=20 \mathrm{~nm}$ ). The variation of conductivity and critical current density $\left(J^{*}\right)$ for the cross-linked nanoparticles at different pore sizes are reported in SI Appendix, Fig. S5. It is seen that $J^{*}$ varies in the range $\sim 5-9 \mathrm{~mA} / \mathrm{cm}^{2}$ for the materials studied. Using the previously reported values of $\mathrm{Li}^{+}$transference number $(0.36)$ and lithium ion diffusivity $\left(3 \times 10^{-6} \mathrm{~cm}^{2} / \mathrm{s}\right)$ for $1 \mathrm{M} \mathrm{EC:DMC-LiPF} 6$ electrolyte, the diffusion-limited current density can be estimated to be $9 \mathrm{~mA} / \mathrm{cm}^{2}$ for the same interelectrode spacing. (46, 47).

Fig. $3 A$ reports snapshots of the negative electrode at different stages of electrodeposition for the cases without any separator, with a commercial glass-fiber separator and cross-linked hairy nanoparticles (r.c.p. pore size $=20 \mathrm{~nm}$ ) at a constant current density of $8 \mathrm{~mA} / \mathrm{cm}^{2}$, which is 0.9 times the $J^{*}$ calculated for liquid electrolyte. Through visual inspection, it is obvious that $\mathrm{Li}$ electrodeposition in the absence of a separator is uneven, mossy, and blackened, which is indicative of the side reactions between electrode and electrolyte as well as the porous nature of the deposited layers. At first appearance, the deposits are already quite large, at least $100 \mu \mathrm{m}$ in diameter, which is much larger than typical $\mathrm{Li}$ dendrite nucleate sizes assumed in the literature (6). Deposition studies based on a commercial glass-fiber separator show that deposition is relatively compact until a capacity of around $2 \mathrm{mAh} / \mathrm{cm}^{2}$ (i.e., $\sim 10 \mu \mathrm{m} / \mathrm{cm}^{2} \mathrm{Li}$ is transferred), where after dendritic structures develop and proliferate in time, ultimately piercing the separator and continue their growth through the separator. This sequence is associated with the shortcircuiting phenomena described in the Introduction and can lead to catastrophic cell failure by fire, explosion, or both. In comparison with the cases with and without separator, the deposition pattern with the cross-linked hairy nanoparticles display uniform deposition for entire $1 \mathrm{~h}$ of the charging process. The accelerated movies of electrodeposition are provided for all of the three cases. Movies S1-S3 are 10-s movies of 1,500-s-long electrodeposition for neat electrolyte, with glass-fiber separator and cross-linked nanoparticles (r.c.p. $=20 \mathrm{~nm}$ ), respectively.

By tracking the advancing front of the Li electrode over time, it is possible to quantify the dendrite growth as a function of time (Fig. $3 B$ ). The apparent growth rate obtained from the initial time through the assumption of linearity is shown in the inset. The larger-sized error bars for the neat electrolyte case is a reflection of the unevenness of electrodeposition. The apparent growth rate of dendrite in the commercial glass-fiber separator is 

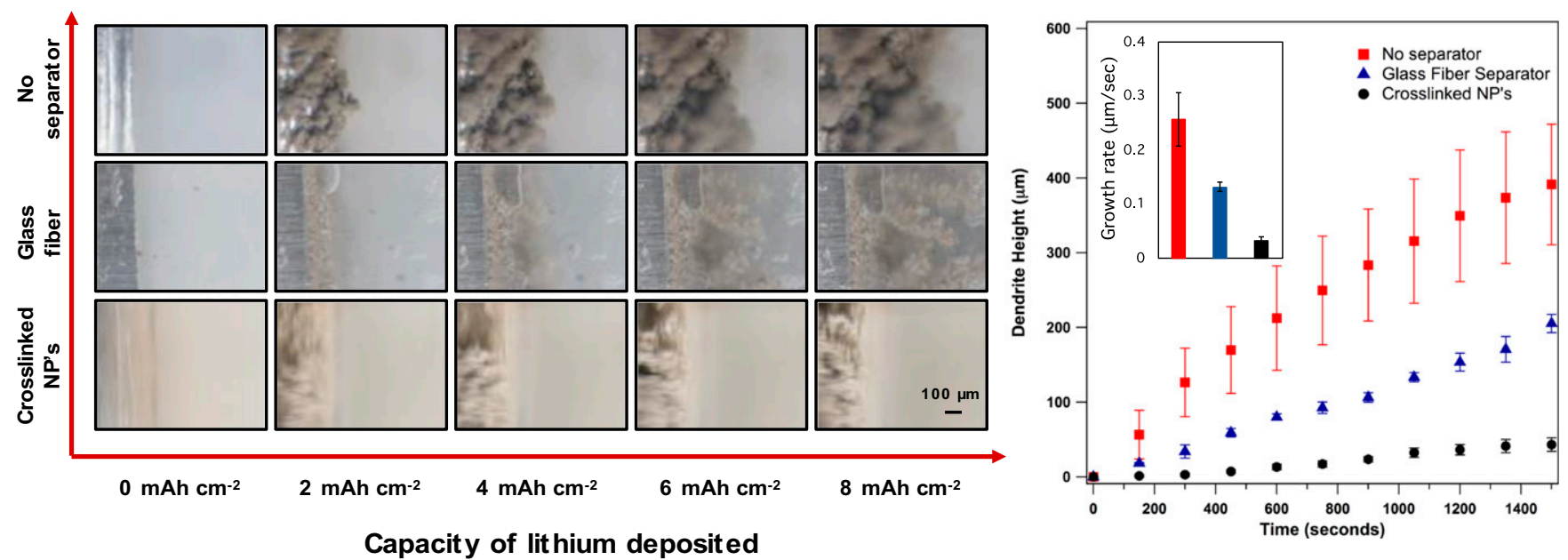

Fig. 3. Stability of electrodeposition by direct visualization of electrode. $(A)$ Snapshots of the electrode and electrolyte every 15 min during charging at the rate of $8 \mathrm{~mA} \mathrm{~cm}^{-2}$. (B) Height of dendrite at various points of the electrode for the initial 1,500 s. (Inset) Comparison of the growth rate by assuming a linear growth for electrodeposition at $8 \mathrm{~mA} \mathrm{~cm}^{-2}$.

calculated to be $\sim 0.13 \mu \mathrm{m} / \mathrm{s}$, while for the cross-linked hairy nanoparticles it is estimated to be $0.03 \mu \mathrm{m} / \mathrm{s}$, which is modestly larger than the theoretical rate $(0.011 \mu \mathrm{m} / \mathrm{s})$ for perfectly smooth electrodeposition at $8 \mathrm{mAh} / \mathrm{cm}^{2}$.

This idea that one could control the stability of electrodeposition by manipulating the nanostructure of an electrolyte (SI Appendix, Fig. S6) was previously discussed in the theoretical analysis by Tikekar et al. $(7,38)$ and has also been adapted in an ad hoc manner to explain previous experimental results $(48,49)$. In this analysis, deposition in membranes with pores larger than the critical nucleate diameter at which electrodeposition is unstable is thought to lead to unchecked growth of dendrites that penetrate through these pores, as in the case of the glass-fiber separator. In contrast, in the cross-linked hairy nanoparticles the pore size of $20 \mathrm{~nm}$ is evidently much smaller than the nucleate size, leading to a significant retardation of dendrite growth. To determine the effect of solid electrolyte interphase formation and chemical instability on the topological features observed during the electrodeposition process, we performed postmortem analysis of the lithium electrode after repeated charge and discharge cycling in a coin cell. Specifically, we utilized a symmetric lithium battery with the cross-linked nanoparticle electrolyte $(20 \mathrm{~nm})$ and operated the battery at a current density of $0.1 \mathrm{~mA} / \mathrm{cm}^{2}$ for $100 \mathrm{~h}$, with each half-cycle comprising $3 \mathrm{~h}$, as shown in SI Appendix, Fig. S7. We intentionally chose low current densities for the operation to isolate the effect of chemical and not morphological instabilities. The SEM image of the extracted lithium metal is seen to be smooth and unperturbed (SI Appendix, Fig. S8). This provides evidence that the observed evolution of electrode surface in visualization experiment is dominant by dendritic growth of lithium and not due to parasitic reactions at the interface.

One could make these tentative ideas more concrete by interrogating the evolution of the interface in cross-linked electrolytes in which the average pore size is systematically varied. SI Appendix, Fig. S9 show the snapshots of deposition at same current density of $8 \mathrm{~mA} / \mathrm{cm}^{2}$, and SI Appendix, Fig. S10 reports the analytical results from such studies in which the morphology of the Li-electrolyte interface is characterized in cross-linked nanoparticles with varying pore size (or, weight fraction of $\mathrm{SiO}_{2}$ in the composite). The liquid electrolyte weight was maintained to be the same in all cases reported in this work, which is twice that of the composite weight. The results show that when the effective pore size of the membrane is $1,000 \mathrm{~nm}$, dendrite growth is unimpeded and rapid. However, at high volume fractions of silica nanoparticles corresponding to interparticle distance of order $500 \mathrm{~nm}$, SI Appendix, Fig. S10 shows two regimes of dendrite growth, wherein the growth rate at the initial time is low, followed by an accelerated rate of dendrite propagation at longer time. The results in SI Appendix, Fig. S9 also show that for the membranes with 500-nm pores, dendrites with "needle-like" morphology are visible at $4 \mathrm{mAh} / \mathrm{cm}^{2}$, in contrast to the mossy-blackened deposited lithium apparent when the pores are larger and in neat electrolytes. Furthermore, it is seen that in membranes with effective pore size of around $100 \mathrm{~nm}$ (Movie S4), the deposition is similarly restricted as for the 20 -nm case, implying that there is a threshold pore size between 500 and $100 \mathrm{~nm}$ where the resistance to dendrite proliferation is large enough to prevent growth.

Fig. 4 summarizes the growth rate deduced from experiments as a function of average membrane pore size and elastic modulus, respectively, at $8 \mathrm{~mA} / \mathrm{cm}^{2}$ as well as for varying current densities such that $J / J^{*}$ remains constant at 0.9 (SI Appendix, Fig. S5). Optical snapshots of the initial deposition and the corresponding current densities $\left(\right.$ at $\left.J / J^{*}=0.9\right)$ are reported in $S I$ Appendix, Fig. S11. The experimental results reveal a sudden transition from stable deposition to dendrite growth between $100-500-n m$ pore size in agreement with our hypothesis that electrodeposition stability is function of electrolyte length scale. Also, the shear modulus measured from rheology does not have a linear relation with growth rate, which can be rationalized by the fact that the cross-linked membrane comprises building
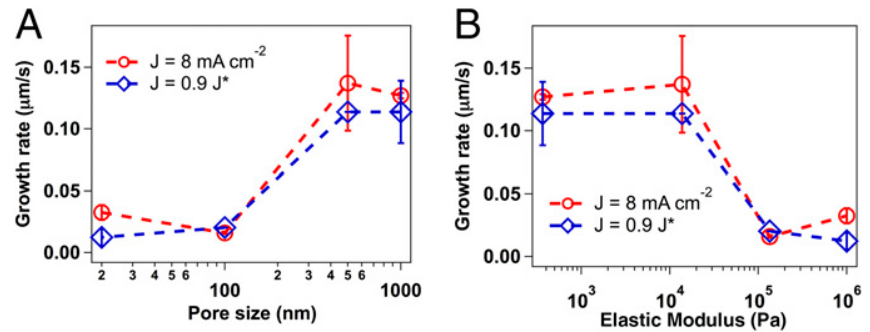

Fig. 4. Analyzing pore-size dependence on dendrite growth. ( $A$ and $B$ ) Growth rate at different pore sizes and corresponding elastic modulus, respectively, for the cross-linked hairy nanoparticles at current density of $8 \mathrm{~mA} / \mathrm{cm}^{2}$ as well as for variable current densities matching 0.9 times of the limiting current densities measured for respective samples. 
blocks having different inherent modulus $\left(\mathrm{SiO}_{2}\right.$ and polymer). As the deposition takes place in the interparticle gap, the growing "dendrites" only experience the modulus of the liquid electrolyte and polymers; thus, the length scale of the polymeric phase plays a greater role than the overall modulus of the material. We also analyze the growth rate of dendrites as a function of pore size obtained from TEM micrograph analysis as well as the pore volume calculated from plateau modulus as $k T / G^{\prime}$. As shown in SI Appendix, Fig. S12, a similar nonlinear behavior is observed.

Fig. $5 A$ plots the perturbation growth rate versus wavelength deduced from the analysis of Tikekar et al. (38). It is seen that the growth rate is negative for small wavelengths and goes through a maximum at a particular wavelength that decreases as the current density approaches critical current density, $J^{*}$. The first of these results can be shown to arise from the greater influence of surface tension in suppression growth of small dendrite nucleates, while the presence of the maximum reflects the effect of the physical (macroscopic) dimensions of the simulation cell in restricting nucleate size below certain limits. As the current density increases, the growth rate of the fastest (most unstable) mode increases and its wavelength decreases because the interfacial tension has to compete with progressively more aggressive electroconvection to stop the roughening. The fastest growing mode can therefore be thought of as representative of the typical size of dendrites that one would observe in an experiment under similar conditions. A porous material would restrict the size of the growing dendrite, since the deposition can only happen on length scales smaller than the pore size. In Fig. $5 B$, we show the calculated growth rate for the fastest growing mode as a function of the critical wavelength. For very large pores, the pore size is predicted to have no effect on the dendrite growth, and the growth occurs more or less at the same rate as in the absence of the porous material. For smaller pores, however, the pore diameter restricts the sizes of the dendrites that can form in them, causing the growth rate of the fastest mode to plummet. Below a certain size, there can be no unstable deposition, as surface tension adequately competes with current-density-driven dendritic growth at all possible length scales. This critical pore size decreases with current density due to the requirement of a higher contribution from surface tension as seen in SI Appendix, Fig. S13. SI Appendix, Fig. S13 (Inset) plots the analogous growth rate deduced from experiments as a function of membrane pore size for a fixed current density, $J / J^{*} \sim 0.9$. The experimental results show a similar trend as calculated from the linear stability analysis, wherein there is sudden transition from stable deposition to dendrite growth. The transition point from the theoretical prediction corresponding to nonzero growth is $\sim 1.6 \mu \mathrm{m}$, while the experiments indicate the cross-over between $100-500$-nm pore size as previously mentioned in Fig. $4 A$. Considering the variety of sources of error in comparing the experiments and theory, including the tortuosity of the pores in
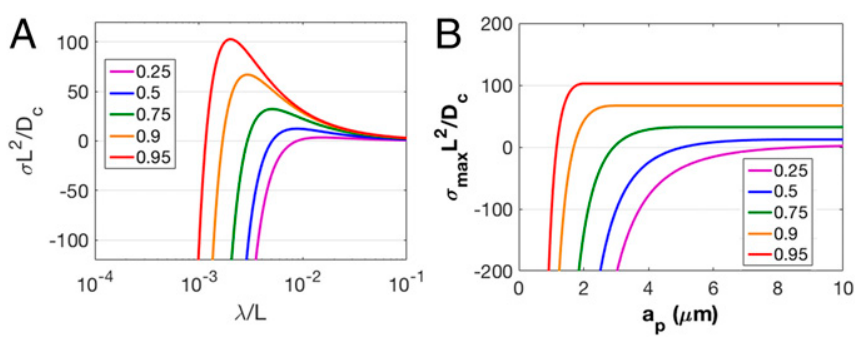

Fig. 5. Linear stability analysis of dendrite growth. (A) Normalized growth rate of the deposited nucleate at different normalized wave numbers obtained by linear stability analysis. $(B)$ Maximum growth at different theoretical pore sizes at various effective currents. The different lines in parts $A$ and $B$ represent different values of $J / J^{*}$. the cross-linked materials, the polydispersity of the particle size, uncertainty in the value of the Li transference number under the conditions of the experiments (48), and the deviation from linearity in calculation of initial growth rate, the agreement between the theoretical and observed transition is fair.

In conclusion, we utilized a cross-linked network by covalently linking polymer-grafted nanoparticles, where varying the volume fraction of the nanoparticles in the composite can conveniently change the effective pore size. It was shown that these crosslinked nanoparticles when used as gel electrolytes showed stable electrodeposition in contrast to mossy and dendritic morphology for cases without separator and with glass fibers, respectively. We performed visualization experiments for different pore sizes determined by volume fractions to demonstrate that below $500 \mathrm{~nm}$, the deposition is smooth and compact, while it is dendritic for other cases. These experimental observations were rationalized using linear stability analysis of dendrite growth at different perturbation length scale representing pore diameter of the electrolytes. It was understood that below the limiting current density, the electrodeposition can be stabilized by implementing a separator with pore size lower than the length scale of the most unstable nucleate. Overall, this work will provide guidelines for designing solid-state electrolytes and separators for metal-based batteries.

\section{Materials and Methods}

Materials. Lithium rods, ethylene carbonate, dimethylene carbonate, lithium hexafluorophosphate, anhydrous copper chloride, dimethyl sulfoxide, glass fibers Ludox SM30 colloidal silica $(\mathrm{d}=10 \pm 2 \mathrm{~nm}$ ), poly(propylene glycol)toluene-2,4-diisocyante were all purchased from Sigma-Aldrich. Hydoxy ter minated poly (ethylene oxide)-silane was obtained from Gelest. Copper foil was obtained from Alfa Aesar. All of the chemicals were used as received after rigorous drying in an $\sim 0$-ppm water level and <5-ppm oxygen glove box.

Linear Stability Analysis. The details regarding the linear stability analysis were presented in a previous paper by Tikekar et al. (38)

Cross-Linked Hairy Nanoparticles Synthesis. The reaction procedure for crosslinking between the grafted silica nanoparticles and functionalized PPO polymer was given in a previous work (18). Different cross-linked nanoparticles with varying pore sizes were synthesized by adjusting the ratio between the silica nanoparticles and the PPO polymer as given in SI Ap pendix, Table S1. The ratio between the liquid electrolyte and the crosslinked composite was kept constant at 2:1 by weight.

Dielectric Spectroscopy. The dielectric spectroscopy measurements were done using a Novocontrol N40 Broadband Dielectric instrument. The cross-linked hairy nanoparticles as well as neat PPO (without liquid electrolyte) were casted at the center of a teflon o-ring. The measurements were done in a frequency range from $10^{-3}$ to $10^{7} \mathrm{~Hz}$ at various temperatures.

TEM. The TEM measurements were performed to understand the nanostructure of the cross-linked hairy nanoparticles using an FEI T12 Spirit TEM. A thin layer of the materials was casted onto the TEM grid using chloroform as the solvent and it was allowed to cross-link in the TEM grid. Before the measurements, the samples were dried overnight at $60^{\circ} \mathrm{C}$.

SEM. SEM analysis was done using the LEO155FESEM instrument. In this ex periment, symmetric lithium coin cell was constructed using the cross-linked electrolyte (r.c.p. = $20 \mathrm{~nm}$ ). "Plate-strip" measurements were performed at a current density of $0.1 \mathrm{~mA} / \mathrm{cm} 2$ for $100 \mathrm{~h}$, with each cycle comprising $3 \mathrm{~h}$ thereafter, the lithium electrode was extracted and dried in the antechamber of a glove box overnight.

Mechanical Properties. The cross-linking process was studied mechanically by casting the precursor materials in the rheometer with parallel-plate geometry of diameter $25 \mathrm{~mm}$ using an ARES Rheometer. Specifically, for time-sweep measurements, a constant strain amplitude of $5 \%$ and fixed frequency of $10 \mathrm{~Hz}$ was applied until the storage modulus reached a fixed value. The frequency-sweep measurements were performed at a fixed strain amplitude of $5 \%$ between 1 and $100 \mathrm{~Hz}$ for all materials. All of the measurements were 
done without any addition of liquid electrolytes and at the same temperature value of $60^{\circ} \mathrm{C}$.

Direct Visualization Experiments. The visualization experiment was carried out for understanding the electrodeposition process with various forms of electrolytes mentioned in the text. In all of the measurements the ratio between the weight of the composite material and liquid electrolyte $(1 \mathrm{M}$

1. Lin D, Liu Y, Cui Y (2017) Reviving the lithium metal anode for high-energy batteries Nat Nanotechnol 12:194-206.

2. Cheng $X B$, et al. (2015) A review of solid electrolyte interphases on lithium meta anode. Adv Sci (Weinh) 3:1500213.

3. Cheng $X-B$, Zhang $R$, Zhao C-Z, Zhang Q (2017) Toward safe lithium metal anode in rechargeable batteries: A review. Chem Rev 117:10403-10473.

4. Ma L, Hendrickson KE, Wei S, Archer LA (2015) Nanomaterials: Science and applications in the lithium-sulfur battery. Nano Today 10:315-338.

5. Zhang W, et al. (2018) Design principles of functional polymer separators for highenergy, metal-based batteries. Small 14:1703001.

6. Wei S, Choudhury S, Tu Z, Zhang K, Archer LA (2017) Electrochemical interphases for high-energy storage using reactive metal anodes. Acc Chem Res 51:80-88.

7. Tikekar MD, Archer LA, Koch DL (2016) Stabilizing electrodeposition in elastic solid electrolytes containing immobilized anions. Sci Adv 2:e1600320.

8. Chazalviel J-N (1990) Electrochemical aspects of the generation of ramified metallic electrodeposits. Phys Rev A 42:7355-7367.

9. Bai P, Li J, Brushett FR, Bazant MZ (2016) Transition of lithium growth mechanisms in liquid electrolytes. Energy Environ Sci 9:3221-3229.

10. Choudhury S, et al. (2017) Electroless formation of hybrid lithium anodes for fast interfacial ion transport. Angew Chem Int Ed Engl 56:13070-13077.

11. Choudhury S, et al. (2017) Designing solid-liquid interphases for sodium batteries. Na Commun 8:898.

12. Wood KN, et al. (2016) Dendrites and pits: Untangling the complex behavior of lithium metal anodes through operando video microscopy. ACS Cent Sci 2:790-801.

13. Zheng G, et al. (2014) Interconnected hollow carbon nanospheres for stable lithium metal anodes. Nat Nanotechnol 9:618-623.

14. Monroe C, Newman J (2004) The effect of interfacial deformation on electrodeposition kinetics. J Electrochem Soc 151:A880.

15. Monroe C, Newman J (2005) The impact of elastic deformation on deposition kinetics at lithium/polymer interfaces. J Electrochem Soc 152:A396.

16. Stone GM, et al. (2012) Resolution of the modulus versus adhesion dilemma in solid polymer electrolytes for rechargeable lithium metal batteries. J Electrochem Soc 159: A222-A227.

17. Agrawal A, Choudhury S, Archer La (2015) A highly conductive, non-flammable polymer-Nanoparticle hybrid electrolyte. RSC Adv 5:20800-20809.

18. Choudhury S, Mangal R, Agrawal A, Archer LA (2015) A highly reversible roomtemperature lithium metal battery based on crosslinked hairy nanoparticles. Nat Commun 6:10101.

19. Lu Y, Korf K, Kambe Y, Tu Z, Archer LA (2014) Ionic-liquid-nanoparticle hybrid electrolytes: Applications in lithium metal batteries. Angew Chem Int Ed Eng/ 53:488-492.

20. Zhang J, Sun B, Huang X, Chen S, Wang G (2014) Honeycomb-like porous gel polyme electrolyte membrane for lithium ion batteries with enhanced safety. Sci Rep 4:6007.

21. Stephan M (2006) Review on gel polymer electrolytes for lithium batteries. Eur Polym J 42:21-42.

22. Wu H, et al. (2013) Stable Li-ion battery anodes by in-situ polymerization of conducting hydrogel to conformally coat silicon nanoparticles. Nat Commun 4:1943.

23. Khurana R, Schaefer JL, Archer LA, Coates GW (2014) Suppression of lithium dendrite growth using cross-linked polyethylene/poly(ethylene oxide) electrolytes: A new approach for practical lithium-metal polymer batteries. J Am Chem Soc 136:7395-7402.

24. Porcarelli L, Gerbaldi C, Bella F, Nair JR (2016) Super soft all-ethylene oxide polymer electrolyte for safe all-solid lithium batteries. Sci Rep 6:19892.

25. Long L, Wang S, Xiao M, Meng Y (2016) Polymer electrolytes for lithium polymer batteries. J Mater Chem A 4:10038-10069.
$\mathrm{LiPF}_{6} \mathrm{EC} / \mathrm{DMC}$ ) was kept constant at 1:2. The details of the visualization cell for lithium deposition measurements were presented in a previous paper (10). The dendrite growth was done using a semiautomatic analysis in MATLAB, where the sizes of the focused dendrites are measured over time.

ACKNOWLEDGMENTS. This work was supported by the National Science Foundation, Division of Materials Research, through Award DMR-1609125.

26. Gurevitch I, et al. (2013) Nanocomposites of titanium dioxide and polystyrene-poly (ethylene oxide) block copolymer as solid-state electrolytes for lithium metal batteries. J Electrochem Soc 160:A1611-A1617.

27. Zhao C-Z, et al. (2017) An anion-immobilized composite electrolyte for dendrite-free lithium metal anodes. Proc Natl Acad Sci USA 114:11069-11074.

28. Tu Z, et al. (2017) Designing artificial solid-electrolyte interphases for single-ion and high-efficiency transport in batteries. Joule 1:394-406.

29. Choudhury S, et al. (2017) Designer interphases for the lithium-oxygen electrochemical cell. Sci Adv 3:e1602809.

30. Ma L, Nath P, Tu Z, Tikekar M, Archer LA (2016) Highly conductive, sulfonated, UVcross-linked separators for Li-S batteries. Chem Mater 28:5147-5154.

31. Lu Y, et al. (2015) Stable cycling of lithium metal batteries using high transference number electrolytes. Adv Energy Mater 5:1402073.

32. Oh $\mathrm{H}$, et al. (2016) Poly(arylene ether)-based single-ion conductors for lithium-ion batteries. Chem Mater 28:188-196.

33. Bouchet $R$, et al. (2013) Single-ion BAB triblock copolymers as highly efficient electrolytes for lithium-metal batteries. Nat Mater 12:452-457.

34. Choudhury S, Archer LA (2016) Lithium fluoride additives for stable cycling of lithium batteries at high current densities. Adv Electron Mater 2:1500246.

35. Lu Y, Tu Z, Archer LA (2014) Stable lithium electrodeposition in liquid and nanoporous solid electrolytes. Nat Mater 13:961-969.

36. Seh ZW, Sun J, Sun Y, Cui Y (2015) A highly reversible room-temperature sodium metal anode. ACS Cent Sci 1:449-455.

37. Zhang X, Cheng X, Chen X, Yan C, Zhang Q (2017) Fluoroethylene carbonate additives to render uniform Li deposits in lithium metal batteries. Adv Funct Mater 27:1605989.

38. Tikekar MD, Archer LA, Koch DL (2014) Stability analysis of electrodeposition across a structured electrolyte with immobilized anions. J Electrochem Soc 161:A847-A855.

39. Choudhury S, Agrawal A, Kim SA, Archer LA (2015) Self-suspended suspensions of covalently grafted hairy nanoparticles. Langmuir 31:3222-3231.

40. Choudhury S, Agrawal A, Wei S, Jeng E, Archer LA (2016) Hybrid hairy nanoparticle electrolytes stabilizing lithium metal batteries. Chem Mater 28:2147-2157.

41. Wei S, et al. (2017) Highly stable sodium batteries enabled by functional ionic polymer membranes. Adv Mater 29:1605512.

42. Stalin S, Choudhury S, Zhang K, Archer LA (2018) Multifunctional cross-linked polymeric membranes for safe, high-performance lithium batteries. Chem Mater 30: 2058-2066.

43. Agarwal P, Kim SA, Archer LA (2012) Crowded, confined, and frustrated: Dynamics of molecules tethered to nanoparticles. Phys Rev Lett 109:258301.

44. Ding Y, et al. (2009) Dielectric spectroscopy investigation of relaxation in C 60-Polyisoprene nanocomposites. Macromol 42:3201-3206.

45. Adachi K, Kotaka T (1993) Dielectric normal mode relaxation. Prog Polym Sci 18: $585-622$

46. Nyman A, Behm M, Lindbergh G (2008) Electrochemical characterisation and modelling of the mass transport phenomena in LiPF6-EC-EMC electrolyte. Electrochim Acta 53:6356-6365.

47. Valøen LO, Reimers JN (2005) Transport properties of LiPF6-based Li-lon battery electrolytes. J Electrochem Soc 152:A882.

48. Tu Z, Nath P, Lu Y, Tikekar MD, Archer LA (2015) Nanostructured electrolytes for stable lithium electrodeposition in secondary batteries. Acc Chem Res 48:2947-2956.

49. Tu Z, et al. (2017) Nanoporous hybrid electrolytes for high-energy batteries based on reactive metal anodes. Adv Energy Mater 7:1602367. 